\title{
Stability and asymptotic behaviour of solutions of the heat equation
}

\author{
MOHAMMED AAssila \\ Institut de Mathématique, Université de Fribourg, Pérolle, CH-1700 Fribourg, \\ Switzerland
}

[Received on 26 November 2001; revised on 12 November 2002]

\begin{abstract}
In this paper we study the boundary stabilization of the heat equation. The stabilization is achieved by applying either Dirichlet or Neumann feedback boundary control. Furthermore, we consider the asymptotic behaviour of the heat equation with general linear delay or nonlinear power time delay. We prove that the energy does not grow faster than a polynomial.
\end{abstract}

Keywords: heat equation; boundary control; stabilization; proportional time delay.

\section{Introduction}

Consider the Cauchy-Dirichlet problem for the porous media equation with source term

$$
\begin{aligned}
& u_{t}-\Delta\left(|u|^{m-1} u\right)-|u|^{p-1} u=0 \quad \text { in } \quad \Omega \times \mathbb{R}_{+} \text {, } \\
& u=0 \quad \text { on } \quad \partial \Omega \times \mathbb{R}_{+}, \\
& u(0)=u_{0} \quad \text { in } \quad \Omega \text {, }
\end{aligned}
$$

where $\Omega$ is a bounded and smooth subset of $\mathbb{R}^{n}, n \geqslant 1, m>0$ and $p \geqslant 1$. Problem (1.1)-(1.3) (see Galaktionov, 1981; Samarskii et al., 1995) describes the propagation of thermal perturbations in a medium with a nonlinear heat conduction coefficient and a heat source depending on the temperature when $u_{0} \geqslant 0$. Local existence for the solutions of (1.1)-(1.3) has been proved when $m>1$ (the so-called slow diffusion case) in Galaktionov (1981), Levine \& Saks (1984), Nakao (1983), Samarskii et al. (1995) and when $0<m<1$ (the fast diffusion case) in Filo (1987). The same type of results holds for the heat equation with source, when $m=1$. See for example Ball (1977), Fujita (1966, 1968), Levine (1973), Tsutsumi (1972). However, other results are known for the heat equation when $1<p \leqslant$ $\frac{n+2}{n-2}$ (the last condition being necessary only when $n \geqslant 3$ ) and $u_{0} \in H_{0}^{1}(\Omega)$.

For large initial data $u_{0}$ in some sense, it is well known that the solution $u$ of (1.1)-(1.3) with $m=1$ blows up in a finite time (see Ikehata \& Suzuki, 2000), meanwhile for small initial data, exponentially decaying solutions are obtained (see Ikehata \& Suzuki, 2000 and the references therein). In a recent paper, Ikehata (2000) showed that all the global solutions for (1.1)-(1.3) with $m=1$ naturally contain a Palais-Smale sequence so that the global compactness result due to Struwe (1984) can be applied to this functional sequence (see also Cerami et al., 1986).

In Section 2 we consider the non-dimensionalized heat equation with boundary 
prespecified at $x=0$ only

$$
\begin{aligned}
u_{t}-u_{x x}-\lambda u & =0 \quad \text { in } \quad(0,1) \times \mathbb{R}_{+}, \\
u_{x}(0, t) & =0 \quad t>0, \\
u(x, 0) & =u_{0}(x) \quad \text { in } \quad(0,1),
\end{aligned}
$$

where the constant $\lambda \geqslant 0$ is a constant parameter.

The system (1.4)-(1.6) is motivated by a physical problem. Indeed, consider the problem of heat conduction in a rod of small cross-section. We assume that each element of the surface of the rod loses heat to a surrounding medium by radiation and in addition, that the heat is generated inside the rod due to a constant electric current flowing through the rod. Let $H$ be the surface conductance (emissivity) of the rod, $i$ the strength of the current and $\rho_{e}$ electrical resistivity. The electric resistivity $\rho_{e}$ changes linearly with the temperature as $\rho_{e}(T)=\rho_{e}\left(T_{1}\right)\left(1-\alpha_{e}\left(T-T_{1}\right)\right)$ where $T_{1}$ stands for the temperature around which the $\rho_{e}$ is linearized, and $\alpha_{e}$ is the thermal coefficient of electric resistivity. The heat equation now becomes (see Carlsaw, 1921, Chapter 4)

$$
T(l, t)_{t}=k T(l, t)_{l l}-v\left(T(l, t)-T_{0}\right)+B\left(1-\alpha_{e}\left(T(l, t)-T_{1}\right)\right),
$$

where $v=\frac{H p}{c \rho A}, B=\frac{i^{2}}{c \rho A^{2}} \rho_{e}\left(T_{1}\right), A$ denotes the cross-section, $p$ the perimeter, $\rho$ the density, $c$ the specific heat and $k$ the diffusivity. Defining the dimensionless length, time and temperature variables as

$$
x=\frac{l}{L}, \quad \tau=\frac{t}{\frac{L^{2}}{k}}, \quad u=\frac{T-T_{e}}{T_{e}},
$$

where $T_{e}=T_{0}+B \frac{\alpha_{e}\left(T-T_{0}\right)-1}{B \alpha_{e}-v}$ stands for the constant equilibrium temperature distribution along the rod and $x \in[0,1]$, we obtain

$$
u_{\tau}(x, \tau)=u_{x x}(x, \tau)+\lambda u(x, \tau),
$$

where $\lambda=\frac{L^{2}\left(B \alpha_{e}-v\right)}{k}$. Depending on the geometry of the rod and the magnitude of the current $i, \lambda$ can be either positive or negative.

Under the Dirichlet boundary condition at $x=1: u(1, t)=0, t>0,(1.4)$ is unstable if $\lambda>\frac{\pi^{2}}{4}$ since for $\lambda=0, \frac{\pi^{2}}{4}$ is the first eigenvalue of

$$
\begin{aligned}
u_{t}-u_{x x}-\lambda u & =0 \quad \text { in } \quad(0,1) \times \mathbb{R}_{+}, \\
u_{x}(0, t) & =0 \quad t>0, \\
u(1, t) & =0 \quad t>0, \\
u(x, 0) & =u_{0}(x) \quad \text { in } \quad(0,1) .
\end{aligned}
$$

A natural question is, if $\lambda>\frac{\pi^{2}}{4}$ can one find a Dirichlet/Neumann boundary feedback law $u(1, t) / u_{x}(1, t)$ that exponentially stabilizes (1.4)-(1.6)? We answer this question positively in Section 2. 
In the second part of the paper (Section 3) we study the asymptotic behaviour of solutions of heat equation with general linear time delay. Kato \& McLeod (1971) discussed the functional-differential equation

$$
u^{\prime}(t)=a u(\lambda t)+b u(t) \quad 0 \leqslant t<\infty
$$

where $a$ is a possibly complex constant, $b$ a real constant and $\lambda$ a non-negative constant. They showed that (1.7) is well posed if $0 \leqslant \lambda \leqslant 1$ and studied the asymptotic properties of solutions as $t \rightarrow+\infty$. delay

In this paper we generalize problem (1.7) to the heat equation with general linear time

$$
\begin{aligned}
u_{t}(x, t)-\varepsilon \Delta u(x, t)-a u(x, \lambda t-\sigma) & =0 & \text { in } & & \Omega \times \mathbb{R}_{+}, \\
u(x, t) & =0 & \text { on } & & \Gamma \times \mathbb{R}_{+}, \\
u(x, s) & =u_{0}(x, s) & & \text { in } & \Omega \times[-\sigma, 0],
\end{aligned}
$$

where $\Omega$ is a bounded domain in $\mathbb{R}^{n}$ with boundary $\Gamma, \varepsilon>0$ denotes the heat conductivity constant, $0<\lambda<1, \sigma \geqslant 0$ are delay parameters and $u_{0}(x, s)$ is an initial state in an appropriate function space. Obviously, (1.8) is very similar to (1.7).

Another possible method of delay is the following power delay (note that the time is in advance before time $t=1$ ):

$$
\begin{array}{rlrlrl}
u_{t}(x, t)-\varepsilon \Delta u(x, t)-a u\left(x, t^{\lambda}\right) & =0 & & \text { in } & & \Omega \times \mathbb{R}_{+}, \\
u(x, t) & =0 & \text { on } & \Gamma \times \mathbb{R}_{+}, \\
u(x, 0) & =u_{0}(x) & & \text { in } & \Omega .
\end{array}
$$

To the best of my knowledge, problem (1.11)-(1.13) has not been studied before. By using ideas from Kato \& McLeod (1971), we shall prove that under certain conditions on $a, \varepsilon$ and $\lambda$, the solutions of (1.8)-(1.10), (1.11)-(1.13) do not grow faster than a polynomial.

The paper is organized as follows. In Section 2, we study the stabilization of (1.4)(1.6). In Section 3, we study the asymptotic behaviour of solutions to (1.8)-(1.10), (1.11)(1.13). Throughout the paper we denote by $\|\cdot\|$ the usual $L^{2}$-norm.

\section{Stabilization}

\subsection{Dirichlet boundary condition}

In order to stabilize exponentially the system (1.4)-(1.6), we choose as Dirichlet boundary feedback law

$$
u(1, t)=-a \tan (a) \int_{0}^{1} u(s, t) \mathrm{d} s
$$

where $a$ is a real number.

Consider the following problem:

$$
\left\{\begin{array}{l}
u_{t}-u_{x x}-\lambda u=0 \quad \text { in } \quad(0,1) \times \mathbb{R}_{+}, \\
u_{x}(0, t)=0, \quad t>0, \\
u(1, t)=-a \tan (a) \int_{0}^{1} u(s, t) \mathrm{d} s, \quad t>0, \\
u(x, 0)=u_{0}(x) .
\end{array}\right.
$$


Then the main result of this section is the following theorem.

Theorem 2.1 Assume that $\lambda \in\left[0, \frac{3 \pi^{2}}{4}\right)$ and $a \in\left(\max \left\{0, \operatorname{sgn}\left(\frac{\lambda}{2}-\frac{\pi^{2}}{8}\right) \sqrt{\left|\frac{\lambda}{2}-\frac{\pi^{2}}{8}\right|}\right\}, \frac{\pi}{2}\right)$, then

(i) for any $u_{0} \in C(0,1)$, problem $\left(P_{1}\right)$ has a unique classical solution $u$ satisfying

$$
\|u(t)\| \leqslant C\left\|u_{0}\right\| \mathrm{e}^{-\left(\frac{\pi^{2}}{4}+2 a^{2}-\lambda\right) t}, \quad \forall t \geqslant 0
$$

where $C$ is a positive constant independent of $u_{0}$,

(ii) for any $u_{0} \in H^{1}(0,1)$, problem $\left(P_{1}\right)$ has a unique strong solution $u$ satisfying

$$
\|u(t)\|_{H^{1}} \leqslant C\left\|u_{0}\right\|_{H^{1}} \mathrm{e}^{-\frac{1}{2}\left(\frac{\pi^{2}}{4}+2 a^{2}-\lambda\right) t}, \quad \forall t \geqslant 0
$$

where $C$ is a positive constant independent of $u_{0}$.

REMARK The main idea in the proof of Theorem 2.1 is the coordinate transformation (2.1) (see below), because once we have (2.1) the procedure to derive decay estimates for (i) and (ii) of Theorem 2.1 is the standard energy method.

Proof. For $x \in[0,1]$ and $0<a<\frac{\pi}{2}$, we introduce the new variable $v$ defined by

$$
v(x, t):=u(x, t)+a \tan (a x) \int_{0}^{x} u(s, t) \mathrm{d} s .
$$

This coordinate transformation has an inverse,

$$
u(x, t)=v(x, t)-a \sin (a x) \int_{0}^{x} \frac{v(s, t)}{\cos (a s)} \mathrm{d} s .
$$

Indeed, for $x=0$ we have $v(0, t)=u(0, t)$, and for $x \in(0,1]$ we have

$$
\begin{gathered}
\left(-a \tan (a x) \int_{0}^{x} u(s, t) \mathrm{d} s\right)_{x}=-a \tan (a x) u(x, t)-\frac{a^{2}}{\cos ^{2}(a x)} \int_{0}^{x} u(s, t) \mathrm{d} s \\
=-a \tan (a x)\left[v(x, t)-a \tan (a x) \int_{0}^{x} u(s, t) \mathrm{d} s\right]-\frac{a^{2}}{\cos ^{2}(a x)}\left(\frac{a \tan (a x)}{a \tan (a x)} \int_{0}^{x} u(s, t) \mathrm{d} s\right) \\
=-a \cot (a x) a \tan (a x) \int_{0}^{x} u(s, t) \mathrm{d} s-a \tan (a x) v(x, t) .
\end{gathered}
$$

Hence we get

$$
\begin{gathered}
-a \tan (a x) \int_{0}^{x} u(s, t) \mathrm{d} s=\int_{0}^{x}\left[-a \tan (a s) v(s, t) \mathrm{e}^{\int_{s}^{x} a \cos (a \eta) \mathrm{d} \eta}\right] \mathrm{d} s \\
=\mathrm{e}^{\ln (\sin (a x))} \int_{0}^{x}\left[-a \tan (a s) v(s, t) \mathrm{e}^{-\ln (\sin (a s))}\right] \mathrm{d} s \\
=-a \sin (a x) \int_{0}^{x} \frac{\tan (a s)}{\sin (a s)} v(s, t) \mathrm{d} s=-a \sin (a x) \int_{0}^{x} \frac{v(s, t)}{\cos (a s)} \mathrm{d} s .
\end{gathered}
$$


Thus

$$
u(x, t)=v(x, t)-a \sin (a x) \int_{0}^{x} \frac{v(s, t)}{\cos (a s)} \mathrm{d} s .
$$

Now, we claim that by this coordinate transformation, problem $\left(P_{1}\right)$ is converted into

$$
\left\{\begin{array}{l}
v_{t}-v_{x x}+\left(-\lambda+2 \frac{a^{2}}{\cos ^{2}(a x)}\right) v=0 \quad \text { in } \quad(0,1) \times \mathbb{R}_{+} \\
v_{x}(0, t)=0, \quad v(1, t)=0, \quad t>0 \\
v(x, 0)=v_{0}(x) \quad \text { in } \quad(0,1)
\end{array}\right.
$$

Indeed, we have

$$
\begin{aligned}
v_{t}(x, t) & =u_{t}(x, t)+a \tan (a x) \int_{0}^{x} u_{t}(s, t) \mathrm{d} s \\
& =u_{x x}(x, t)+\lambda u(x, t)+a \tan (a x) \int_{0}^{x}\left(u_{s s}(s, t)+\lambda u(s, t)\right) \mathrm{d} s \\
& =u_{x x}(x, t)+\lambda u(x, t)+a \tan (a x) u_{x}(x, t)+\lambda a \tan (a x) \int_{0}^{x} u(s, t) \mathrm{d} s, \\
v_{x x}(x, t) & =u_{x x}(x, t)+(a \tan (a x))^{\prime \prime} \int_{0}^{x} u(s, t) \mathrm{d} s+2(a \tan (a x))^{\prime} u(x, t) \\
& +a \tan (a x) u_{x}(x, t) .
\end{aligned}
$$

Hence $v_{t}-v_{x x}+\left(-\lambda+2 \frac{a^{2}}{\cos ^{2}(a x)}\right) v=0$ if and only if

$$
\begin{gathered}
{\left[\lambda-2(a \tan (a x))^{\prime}+\left(-\lambda+2 \frac{a^{2}}{\cos ^{2}(a x)}\right)\right] u(x, t)} \\
+\left[(-a \tan (a x))^{\prime \prime}+\lambda a \tan (a x)+\left(-\lambda+2 \frac{a^{2}}{\cos ^{2}(a x)}\right) a \tan (a x)\right] \int_{0}^{x} u(s, t) \mathrm{d} s=0 .
\end{gathered}
$$

A simple computation shows that

$$
\begin{gathered}
\lambda-2(a \tan (a x))^{\prime}+\left(-\lambda+2 \frac{a^{2}}{\cos ^{2}(a x)}\right)=0 \\
(-a \tan (a x))^{\prime \prime}+\lambda a \tan (a x)+\left(-\lambda+2 \frac{a^{2}}{\cos ^{2}(a x)}\right) a \tan (a x)=0 .
\end{gathered}
$$

For the boundary conditions, by differentiating (2.1) and taking $u_{x}(0, t)=0$ we obtain $v_{x}(0, t)=0$, and by substituting $x=1$ in $(2.1)$ and considering the fact that $v(1, t)=0$, we deduce that $\left(P_{1}\right)$ and $\left(\widetilde{P}_{1}\right)$ are equivalent.

It can be shown that the system $\left(\widetilde{P}_{1}\right)$ is exponentially stable if

$$
\min _{0 \leqslant x \leqslant 1}\left(-\lambda+2 \frac{a^{2}}{\cos ^{2}(a x)}\right)=-\lambda+2 a^{2}>-\frac{\pi^{2}}{4} .
$$


This condition is easily verified under the conditions on $\lambda$ and $a$ stated in Theorem 2.1 .

Since $\left(\widetilde{P}_{1}\right)$ is well posed (see Ladyzhenskaya et al., 1968, Chapter 4 ) and the transformation (4.1) is invertible then $\left(P_{1}\right)$ is well posed and by $(2.2)$ there exists a positive constant $c_{1}>0$ such that

$$
\begin{aligned}
\|u(t)\|_{L^{2}} & \leqslant c_{1}\|v(t)\|_{L^{2}}, \\
\|u(t)\|_{H^{1}} & \leqslant c_{1}\|v(t)\|_{H^{1}},
\end{aligned}
$$

and by (2.1) there exists a positive constant $c_{2}>0$ such that

$$
\begin{aligned}
\left\|v_{0}\right\|_{L^{2}} & \leqslant c_{2}\left\|u_{0}\right\|_{L^{2}}, \\
\left\|v_{0}\right\|_{H^{1}} & \leqslant c_{2}\left\|u_{0}\right\|_{H^{1}} .
\end{aligned}
$$

Consequently, it suffices to prove the estimates in (i)-(ii) for the solution $v$ of $\left(\widetilde{P}_{1}\right)$.

(i) We define the energy of a solution $v$ by

$$
E(v(t))=\frac{1}{2} \int_{0}^{1} v^{2}(x, t) \mathrm{d} x .
$$

We have

$$
0=\int_{0}^{1} v\left(v_{t}-v_{x x}+\left(-\lambda+2 \frac{a^{2}}{\cos ^{2}(a x)}\right) v\right) \mathrm{d} x
$$

and the integration by parts yields

$$
E_{t}(v(t))=-\int_{0}^{1} v_{x}^{2}(x, t) \mathrm{d} x-\int_{0}^{1}\left(-\lambda+2 \frac{a^{2}}{\cos ^{2}(a x)}\right) v^{2}(x, t) \mathrm{d} x
$$

Since the operator $-\frac{\partial^{2}}{\partial x^{2}}$ with boundary conditions $u_{x}(0, t)=u(1, t)=0$ has $\frac{\pi^{2}}{4}$ as the smallest eigenvalue, we have

$$
\frac{\pi^{2}}{4}\|v\|^{2} \leqslant\left\|v_{x}\right\|^{2}
$$

and hence

$$
E_{t}(v(t)) \leqslant-2\left(\frac{\pi^{2}}{4}+2 a^{2}-\lambda\right) E(v(t))
$$

Consequently

$$
E(v(t)) \leqslant E(v(0)) \mathrm{e}^{-2\left(\frac{\pi^{2}}{4}+2 a^{2}-\lambda\right) t}, \quad \forall t \geqslant 0
$$

(ii) Define

$$
\mathcal{E}(t)=\int_{0}^{1} v_{x}^{2}(x, t) \mathrm{d} x
$$

By (2.3) we have

$$
E_{t}(t)+\mathcal{E}(t) \leqslant c E(t)
$$

where $c$ denotes various positive constants which may be different at different steps. 
Now, we have

$$
\mathrm{e}^{\left(\frac{\pi^{2}}{4}+2 a^{2}-\lambda\right) t}\left(E_{t}(t)+\mathcal{E}(t)\right) \leqslant c \mathrm{e}^{\left(\frac{\pi^{2}}{4}+2 a^{2}-\lambda\right) t} E(t)
$$

and hence we get

$$
\begin{aligned}
\frac{\mathrm{d}}{\mathrm{d} t}\left(\mathrm{e}^{\left(\frac{\pi^{2}}{4}+2 a^{2}-\lambda\right) t} E(t)\right)+\mathrm{e}^{\left(\frac{\pi^{2}}{4}+2 a^{2}-\lambda\right)} \mathcal{E}(t) & \leqslant\left[\left(\frac{\pi^{2}}{4}+2 a^{2}-\lambda\right)+c\right] \mathrm{e}^{\left(\frac{\pi^{2}}{4}+2 a^{2}-\lambda\right) t} E(t) \\
& \leqslant\left[\left(\frac{\pi^{2}}{4}+2 a^{2}-\lambda\right)+c\right] \mathrm{e}^{-\left(\frac{\pi^{2}}{4}+2 a^{2}-\lambda\right) t} .
\end{aligned}
$$

The integration over $(0, t)$ yields

$$
\mathrm{e}^{\left(\frac{\pi^{2}}{4}+2 a^{2}-\lambda\right) t} E(t)+\int_{0}^{t} \mathrm{e}^{\left(\frac{\pi^{2}}{4}+2 a^{2}-\lambda\right) s} \mathcal{E}(s) \mathrm{d} s \leqslant c E(0) .
$$

We have

$$
0=\int_{0}^{1} v_{x x}\left(v_{t}-v_{x x}+\left(-\lambda+2 \frac{a^{2}}{\cos ^{2}(a x)}\right) v\right) \mathrm{d} x
$$

and hence we get

$$
\begin{aligned}
\mathcal{E}_{t}(t) & =-2 \int_{0}^{1} v_{x x}^{2} \mathrm{~d} x+2 \int_{0}^{1}\left(-\lambda+2 \frac{a^{2}}{\cos ^{2}(a x)}\right) v v_{x x} \mathrm{~d} x \\
& \leqslant-2 \int_{0}^{1} v_{x x}^{2} \mathrm{~d} x+\int_{0}^{1} v_{x x}^{2} \mathrm{~d} x+c \int_{0}^{1} v^{2} \mathrm{~d} x \\
& \leqslant c \int_{0}^{1} v^{2} \mathrm{~d} x \\
& \leqslant c E(t),
\end{aligned}
$$

which implies that

$$
\frac{\mathrm{d}}{\mathrm{d} t}\left(\mathcal{E}(t) \mathrm{e}^{\left(\frac{\pi^{2}}{4}+2 a^{2}-\lambda\right) t}\right) \leqslant c[E(t)+\mathcal{E}(t)] \mathrm{e}^{\left(\frac{\pi^{2}}{4}+2 a^{2}-\lambda\right) t}
$$

Integration from 0 to $t$ yields

$$
\mathcal{E}(t) \mathrm{e}^{\left(\frac{\pi^{2}}{4}+2 a^{2}-\lambda\right) t} \leqslant c[\mathcal{E}(0)+E(0)],
$$

whence

$$
\|u(t)\|_{H^{1}} \leqslant C\left\|u_{0}\right\|_{H^{1}} \mathrm{e}^{-\frac{1}{2}\left(\frac{\pi^{2}}{4}+2 a^{2}-\lambda\right) t}, \quad \forall t \geqslant 0 .
$$




\subsection{Neumann boundary condition}

Equation (1.4) with Neumann boundary condition at $x=1: u_{x}(1, t)=0, t>0$, is unstable for $\lambda>0$.

In order to stabilize exponentially the system (1.4)-(1.6), we choose as Neumann boundary feedback law

$$
u_{x}(1, t)=-(\alpha+a \tan (a)) u(1, t)-\left(\alpha a \tan (a)+\frac{a^{2}}{\cos ^{2} a}\right) \int_{0}^{1} u(s, t) \mathrm{d} s .
$$

Consider the following problem:

$\left\{\begin{array}{l}u_{t}-u_{x x}-\lambda u=0 \quad \text { in }(0,1) \times \mathbb{R}_{+}, \\ u_{x}(0, t)=0, \quad t>0 \\ u_{x}(1, t)=-(\alpha+a \tan (a)) u(1, t)-\left(\alpha a \tan (a)+\frac{a^{2}}{\cos ^{2} a}\right) \int_{0}^{1} u(s, t) \mathrm{d} s, t>0 \\ u(x, 0)=u_{0}(x) \quad \text { in }(0,1) .\end{array}\right.$

The main result of this section is the following theorem.

THEOREM 2.2 Assume that $\lambda \in\left[0,1+\frac{\pi^{2}}{2}\right), a \in\left(\max \left\{0, \operatorname{sgn}\left(\frac{\lambda}{2}-\frac{1}{2}\right) \sqrt{\left|\frac{\lambda}{2}-\frac{1}{2}\right|}\right\}, \frac{\pi}{2}\right)$ and $\alpha>2$. Then,

(i) for any $u_{0} \in C(0,1)$, problem $\left(P_{2}\right)$ has a unique classical solution $u$ satisfying

$$
\|u(t)\| \leqslant C\left\|u_{0}\right\| \mathrm{e}^{-\left(1+2 a^{2}-\lambda\right) t}, \quad \forall t \geqslant 0
$$

where $C$ is a positive constant independent of $u_{0}$,

(ii) for any $u_{0} \in H^{1}(0,1)$, problem $\left(P_{2}\right)$ has a unique strong solution $u$ satisfying

$$
\|u(t)\|_{H^{1}} \leqslant C\left\|u_{0}\right\|_{H^{1}} \mathrm{e}^{-\frac{1}{2}\left(1+2 a^{2}-\lambda\right) t}, \quad \forall t \geqslant 0
$$

where $C$ is a positive constant independent of $u_{0}$.

Proof. The proof is similar to that of Theorem 2.1. Instead of $\left(\widetilde{P}_{1}\right)$ we consider

$$
\left\{\begin{array}{l}
v_{t}-v_{x x}+\left(-\lambda+2 \frac{a^{2}}{\cos ^{2}(a x)}\right) v=0 \quad \text { in } \quad(0,1) \times \mathbb{R}_{+}, \\
v_{x}(0, t)=0, \quad v_{x}(1, t)=-\alpha v(1, t) \quad t>0, \\
v(x, 0)=v_{0}(x) \text { in }(0,1) .
\end{array}\right.
$$

It can be shown that $\left(\widetilde{P}_{2}\right)$ is exponentially stable if

$$
\min _{0 \leqslant x \leqslant 1}\left(-\lambda+2 \frac{a^{2}}{\cos ^{2}(a x)}\right)>-1 .
$$

The relation (2.3) becomes

$$
E_{t}(v(t))=-\alpha v^{2}(1, t)-\int_{0}^{1} v_{x}^{2}(x, t) \mathrm{d} x-\int_{0}^{1}\left(-\lambda+2 \frac{a^{2}}{\cos ^{2}(a x)}\right) v^{2}(x, t) \mathrm{d} x .
$$


Since $v(x, t)=v(1, t)-\int_{x}^{1} v_{s}(s, t) \mathrm{d} s$ we have

$$
\begin{aligned}
\int_{0}^{1} v^{2}(x, t) \mathrm{d} x & \leqslant 2 v^{2}(1, t)+2 \int_{0}^{1}(1-x) \mathrm{d} x \int_{0}^{1} v_{x}^{2}(x, t) \mathrm{d} x \\
& \leqslant 2 v^{2}(1, t)+\int_{0}^{1} v_{x}^{2}(x, t) \mathrm{d} x
\end{aligned}
$$

and consequently

$$
E_{t}(v(t)) \leqslant(2-\alpha) v^{2}(1, t)-2\left(1+2 a^{2}-\lambda\right) E(v(t))
$$

which implies

$$
E(v(t)) \leqslant E(v(0)) \mathrm{e}^{-2\left(1+2 a^{2}-\lambda\right) t} \quad \forall t \geqslant 0
$$

The remainder is the same except with $\frac{\pi^{2}}{4}+2 a^{2}-\lambda$ replaced by $1+2 a^{2}-\lambda$ and $\mathcal{E}$ by

$$
\mathcal{E}(t)=\alpha v^{2}(1, t)+\int_{0}^{1} v_{x}^{2}(x, t) \mathrm{d} x .
$$

\section{Asymptotic behaviour}

3.1 Asymptotic behaviour of solutions to (1.8)-(1.10)

In this section we study the problem (1.8)-(1.10). First we have the following result on the well posedness.

THEOREM 3.1 Assume that $\varepsilon>0,0<\lambda<1$ and $a$ is a real number. Then

(i) for any $T>0$ and $u_{0} \in C\left([-\sigma, 0], L^{2}(\Omega)\right)$, problem (1.8)-(1.10) has a unique mild solution $u$ verifying

$$
u \in C\left([0, T], L^{2}(\Omega)\right),
$$

(ii) for any $T>0$ and $u_{0} \in C^{1}\left([-\sigma, 0], H^{2}(\Omega) \cap H_{0}^{1}(\Omega)\right)$, problem (1.8)-(1.10) has a unique classical solution $u$ verifying

$$
u \in C\left([0, T], H^{2}(\Omega) \cap H_{0}^{1}(\Omega)\right) \cap C^{1}\left([0, T], L^{2}(\Omega)\right) .
$$

Proof. (i) Define the linear operator $A$ by

$$
A u=\varepsilon \Delta u
$$

with domain $D(A)=H^{2}(\Omega) \cap H_{0}^{1}(\Omega)$. It is well known that $A$ generates an analytic semigroup $\mathrm{e}^{t A}$ on $L^{2}(\Omega)$. Then, problem (1.8)-(1.10) can be transformed into the following integral equation:

$$
\begin{aligned}
& u(t)=u_{0}(t), \quad-\sigma \leqslant t \leqslant 0, \\
& u(t)=\mathrm{e}^{t A} u_{0}+a \int_{0}^{t} \mathrm{e}^{A(t-s)} u(\lambda s-\sigma) \mathrm{d} s, \quad t>0 .
\end{aligned}
$$


For any $T>0$ and $u_{0} \in C\left([-\sigma, 0], L^{2}(\Omega)\right)$, the solution $u$ of (3.1)-(3.2) with $u \in$ $C\left([0, T], L^{2}(\Omega)\right)$ is called a mild solution of (1.8)-(1.10).

The existence and uniqueness can be proved in the usual way by Picard's iteration method of successive approximations. The reader is referred to $\mathrm{Wu}$ (1996, Chapter 2) for more details.

(ii) Set $v=u_{t}$. Then $v$ satisfies (1.8)-(1.10) with $a$ replaced by $a \lambda$ and the initial condition $u_{0}(x, s)$ replaced by $v_{0}(x, s)=\left(u_{0}\right)_{s}(x, s) \in C\left([-\sigma, 0], L^{2}(\Omega)\right)$. Hence, by (i) we have

$$
v=u_{t} \in C\left([0, T], L^{2}(\Omega)\right)
$$

and then, by the elliptic regularity we deduce that

$$
u \in C\left([0, T], H^{2}(\Omega) \cap H_{0}^{1}(\Omega)\right) \cap C^{1}\left([0, T], L^{2}(\Omega)\right) .
$$

Now, we study the asymptotic behaviour of the global solution to (1.8)-(1.10). We use some techniques from Kato \& McLeod (1971) to prove that the solutions to problem (1.8)-(1.10) do not grow faster than a polynomial. We have the following result.

THEOREM 3.2 Let $\mu_{0}$ be the smallest eigenvalue of $-\Delta$ with Dirichlet boundary condition. Assume that $\varepsilon>0,0<\lambda<1$ and $a$ is a real number. Then, there exists a positive constant $C=C(\varepsilon, a, \lambda)$ such that the solution of (1.8)-(1.10) satisfies

$$
\begin{gathered}
\|u(t)\| \leqslant C\left\|u_{0}\right\|_{C\left([-\sigma, 0], L^{2}(\Omega)\right)}(1+t)^{\frac{1}{\ln \lambda} \ln \left(\frac{\varepsilon \mu_{0}}{|a|}\right)}, \quad \forall t \geqslant 0, \\
\left\|u_{t}(t)\right\| \leqslant C\left\|u_{0}\right\|_{C^{1}\left([-\sigma, 0], L^{2}(\Omega)\right)}(1+t)^{\frac{1}{\ln \lambda} \ln \left(\frac{\varepsilon \mu_{0}}{|a|}\right)-1}, \quad \forall t \geqslant 0 .
\end{gathered}
$$

Proof. We make the change of variables

$$
t=\mathrm{e}^{s}-\frac{\sigma}{1-\lambda}, \quad \tau=\ln \lambda<0, \quad v(x, s)=\mathrm{e}^{-p s} u\left(x, \mathrm{e}^{s}-\frac{\sigma}{1-\lambda}\right),
$$

where $p=\frac{1}{\ln \lambda} \ln \left(\frac{\varepsilon \mu_{0}}{|a|}\right)$.

The function $v$ satisfies

$$
\left\{\begin{array}{l}
v_{s}(x, s)-\varepsilon \mathrm{e}^{s} \Delta v(x, s)+p v(x, s)-a \mathrm{e}^{p \tau} \mathrm{e}^{s} v(x, \tau+s)=0 \quad \text { in } \quad \Omega \times \mathbb{R} \\
v(0, s)=v(1, s)=0 \quad \text { in } \quad \mathbb{R} .
\end{array}\right.
$$

Indeed, we have

$$
\begin{aligned}
u_{t}(x, t) & =\left(v_{s}(x, s)+p v(x, s)\right) \mathrm{e}^{p s+s} \\
u(x, \lambda t-\sigma) & =u\left(x, \lambda \mathrm{e}^{s}-\frac{\lambda \sigma}{1-\lambda}-\sigma\right) \\
& =u\left(x, \mathrm{e}^{\tau+s}-\frac{\sigma}{1-\lambda}\right) \\
& =\mathrm{e}^{p(\tau+s)} v(x, \tau+s) .
\end{aligned}
$$


Hence, to prove (3.3) it suffices to prove the existence of a positive constant $C=C(\varepsilon, a, \lambda)$ such that the solution $v$ of (3.5) satisfies

$$
\|v(s)\| \leqslant C\left\|u_{0}\right\|_{C\left([-\sigma, 0], L^{2}(\Omega)\right)}, \quad \forall s \geqslant \ln \frac{\sigma}{1-\lambda} .
$$

To prove (3.6) it suffices to prove that

$$
\begin{aligned}
& M_{n} \leqslant C(\varepsilon, a, \lambda) M_{0} \quad n=1,2, \ldots, \\
& M_{0} \leqslant C(\varepsilon, a, \lambda)\left\|u_{0}\right\|_{C\left([-\sigma, 0], L^{2}(\Omega)\right)},
\end{aligned}
$$

where we set

$$
\begin{aligned}
s_{0} & =\ln \frac{\sigma}{1-\lambda}, \\
M_{n} & =\max _{s_{0}-n \tau \leqslant s \leqslant s_{0}-n \tau-\tau}\|v(s)\|, \quad n=0,1, \ldots .
\end{aligned}
$$

By noting that $\mu_{0}\|v(x, t)\|^{2} \leqslant\|\nabla v(t)\|^{2}$ we have by integration by parts

$$
\begin{aligned}
\frac{\mathrm{d}}{\mathrm{d} s} \int_{\Omega} v^{2}(s) \mathrm{d} x & =2 \int_{\Omega} v(s) v_{s}(s) \mathrm{d} x \\
& =2 \varepsilon \mathrm{e}^{s} \int_{\Omega} v(s) \Delta v(s) \mathrm{d} x-2 p \int_{\Omega} v^{2}(s) \mathrm{d} x-2 a \mathrm{e}^{p \tau} \mathrm{e}^{s} \int_{\Omega} v(s) v(s+\tau) \mathrm{d} x \\
& =-2 \varepsilon \mathrm{e}^{s} \int_{\Omega}|\nabla v(s)|^{2} \mathrm{~d} x-2 p \int_{\Omega} v^{2}(s) \mathrm{d} x-2 a \mathrm{e}^{p \tau} \mathrm{e}^{s} \int_{\Omega} v(s) v(s+\tau) \mathrm{d} x \\
& \leqslant-2\left(p+\varepsilon \mu_{0} \mathrm{e}^{s}\right) \int_{\Omega} v^{2}(s) \mathrm{d} x-2 a \mathrm{e}^{p \tau} \mathrm{e}^{s} \int_{\Omega} v(s) v(s+\tau) \mathrm{d} x
\end{aligned}
$$

and hence

$$
\frac{\mathrm{d}}{\mathrm{d} s}\left(\mathrm{e}^{2 p s+2 \varepsilon \mu_{0} e^{s}} \int_{\Omega} v^{2}(s) \mathrm{d} x\right) \leqslant-2 a \mathrm{e}^{p \tau+(2 p+1) s+2 \varepsilon \mu_{0} \mathrm{e}^{s}} \int_{\Omega} v(s) v(s+\tau) \mathrm{d} x .
$$

The integration of (3.11) over $\left(s_{0}-(n+1) \tau, s\right)$ yields

$$
\begin{aligned}
& \exp \left(2 p s+2 \varepsilon \mu_{0} \mathrm{e}^{s}\right) \int_{\Omega} v^{2}(s) \mathrm{d} x \\
& \leqslant \exp \left(2 p\left(s_{0}-(n+1) \tau\right)+2 \varepsilon \mu_{0} \exp \left(s_{0}-(n+1) \tau\right)\right) \int_{\Omega} v^{2}\left(s_{0}-(n+1) \tau\right) \mathrm{d} x \\
& \quad-2 a \int_{s_{0}-(n+1) \tau}^{s} \exp \left[p \tau+(2 p+1) t+2 \varepsilon \mu_{0} \mathrm{e}^{t}\right] \int_{\Omega} v(t) v(t+\tau) \mathrm{d} x \mathrm{~d} t \\
& \leqslant M_{n}^{2} \exp \left[2 p\left(s_{0}-(n+1) \tau\right)+2 \varepsilon \mu_{0} \exp \left(s_{0}-(n+1) \tau\right)\right] \\
& \quad+2|a| M_{n} M_{n+1} \int_{s_{0}-(n+1) \tau}^{s} \exp \left[p \tau+(2 p+1) t+2 \varepsilon \mu_{0} \mathrm{e}^{t}\right] \mathrm{d} t .
\end{aligned}
$$


On the other hand, we have

$$
\begin{aligned}
& \int_{s_{0}-(n+1) \tau}^{s} \exp \left[(2 p+1) t+2 \varepsilon \mu_{0} \mathrm{e}^{t}\right] \mathrm{d} t \\
&=\left[\frac{\mathrm{e}^{t}}{2 p+2 \varepsilon \mu_{0} \mathrm{e}^{t}} \exp \left(2 p t+2 \varepsilon \mu_{0} \mathrm{e}^{t}\right)\right]_{s_{0}-(n+1) \tau}^{s} \exp \left(2 p t+2 \varepsilon \mu_{0} \mathrm{e}^{t}\right) \frac{d}{d t}\left(\frac{\mathrm{e}^{t}}{2 p+2 \varepsilon \mu_{0} \mathrm{e}^{t}}\right) \mathrm{d} t \\
&-\int_{s_{0}-(n+1) \tau}^{s} \\
& \leqslant {\left[\frac{\mathrm{e}^{t}}{2 p+2 \varepsilon \mu_{0} \mathrm{e}^{t}} \exp \left(2 p t+2 \varepsilon \mu_{0} \mathrm{e}^{t}\right)\right]_{s_{0}-(n+1) \tau}^{s} } \\
&+2|p| \exp \left(2 p s+2 \varepsilon \mu_{0} \mathrm{e}^{s}\right) \int_{s_{0}-(n+1) \tau}^{s} \frac{\mathrm{e}^{t}}{\left(2 p+2 \varepsilon \mu_{0} \mathrm{e}^{t}\right)^{2}} \mathrm{~d} t \\
&= {\left[\frac{\mathrm{e}^{t}}{2 p+2 \varepsilon \mu_{0} \mathrm{e}^{t}} \exp \left(2 p t+2 \varepsilon \mu_{0} \mathrm{e}^{t}\right)\right]_{s_{0}-(n+1) \tau}^{s} } \\
&-\frac{|p|}{\varepsilon \mu_{0}} \exp \left(2 p s+2 \varepsilon \mu_{0} \mathrm{e}^{s}\right)\left[\frac{1}{2 p+2 \varepsilon \mu_{0} \mathrm{e}^{t}}\right]_{s_{0}-(n+1) \tau}^{s} \\
& \leqslant \frac{\mathrm{e}^{s} \exp \left(2 p s+2 \varepsilon \mu_{0} \mathrm{e}^{s}\right)}{2 p+2 \varepsilon \mu_{0} \mathrm{e}^{s}}+\frac{|p| \exp \left(2 p s+2 \varepsilon \mu_{0} \mathrm{e}^{s}\right)}{\varepsilon \mu_{0}\left(2 p+2 \varepsilon \mu_{0} \exp \left(s_{0}-(n+1) \tau\right)\right)} \\
&-\frac{\exp \left(s_{0}-(n+1) \tau\right)}{2 p+2 \varepsilon \mu_{0} \exp \left(s_{0}-(n+1) \tau\right)} \exp \left[2 p\left(s_{0}-(n+1) \tau\right)+2 \varepsilon \mu_{0} \exp \left(s_{0}-(n+1) \tau\right)\right] .
\end{aligned}
$$

Consequently, we deduce from (3.12) that

$$
\begin{aligned}
& \exp \left(2 p s+2 \varepsilon \mu_{0} \mathrm{e}^{s}\right) \int_{\Omega} v^{2}(s) \mathrm{d} x \\
& \leqslant M_{n}^{2} \exp \left[2 p\left(s_{0}-(n+1) \tau\right)+2 \varepsilon \mu_{0} \exp \left(s_{0}-(n+1) \tau\right)\right] \\
& \quad-\frac{2|a| M_{n} M_{n+1} \exp \left(p \tau+s_{0}-(n+1) \tau\right)}{2 p+2 \varepsilon \mu_{0} \exp \left(s_{0}-(n+1) \tau\right)} \\
& \quad \times \exp \left[2 p\left(s_{0}-(n+1) \tau\right)+2 \varepsilon \mu_{0} \exp \left(s_{0}-(n+1) \tau\right)\right] \\
& +2|a| \mathrm{e}^{p \tau} M_{n} M_{n+1} \frac{\mathrm{e}^{s} \exp \left(2 p s+2 \varepsilon \mu_{0} \mathrm{e}^{s}\right)}{2 p+2 \varepsilon \mu_{0} \mathrm{e}^{s}} \\
& +2|a| \mathrm{e}^{p \tau} M_{n} M_{n+1} \frac{|p| \exp \left(2 p s+2 \varepsilon \mu_{0} \mathrm{e}^{s}\right)}{\varepsilon \mu_{0}\left(2 p+2 \varepsilon \mu_{0} \exp \left(s_{0}-(n+1) \tau\right)\right)}
\end{aligned}
$$

Now, we prove that

$$
M_{n+1} \leqslant\left(1+\sigma_{n}\right) M_{n}
$$

with

$$
\begin{aligned}
\sigma_{n} & =1-f\left(s_{0}-(n+1) \tau\right)+\frac{2|a p| \mathrm{e}^{p \tau}}{\varepsilon \mu_{0}\left(2 p+2 \varepsilon \mu_{0} \exp \left(s_{0}-(n+1) \tau\right)\right)}, \\
f(s) & =\frac{2|a| \mathrm{e}^{p \tau} \mathrm{e}^{s}}{2 p+2 \varepsilon \mu_{0} e^{s}} .
\end{aligned}
$$


First, note that if $M_{n+1} \leqslant M_{n}$ then (3.15) holds. So, assume that $M_{n+1}>M_{n}$. Since $f(s)$ defined by (3.17) is increasing on $[0, \infty)$ it follows that $f(s) \leqslant \frac{|a| \mathrm{e}^{p \tau}}{\varepsilon \mu_{0}}=1$. Whence, from (3.14) we deduce that

$$
\begin{aligned}
\int_{\Omega} v^{2}(s) \mathrm{d} x \leqslant & M_{n} M_{n+1}\left[1-f\left(s_{0}-(n+1) \tau\right)\right] \\
& \times \exp \left[2 p\left(s_{0}-(n+1) \tau\right)+2 \varepsilon \mu_{0} \exp \left(s_{0}-(n+1) \tau\right)\right] \exp \left(-2 p s-2 \varepsilon \mu_{0} \mathrm{e}^{s}\right) \\
& +\frac{2|a| \mathrm{e}^{p \tau} M_{n} M_{n+1} \mathrm{e}^{s}}{2 p+2 \varepsilon \mu_{0} \mathrm{e}^{s}}+\frac{2|a p| \mathrm{e}^{p \tau} M_{n} M_{n+1}}{\varepsilon \mu_{0}\left(2 p+2 \varepsilon \mu_{0} \exp \left(s_{0}-(n+1) \tau\right)\right)} \\
\leqslant & M_{n} M_{n+1}\left[1-f\left(s_{0}-(n+1) \tau\right)\right] \\
& +\frac{|a| \mathrm{e}^{p \tau} M_{n} M_{n+1}}{\varepsilon \mu_{0}}+\frac{|a p| \mathrm{e}^{p \tau} M_{n} M_{n+1}}{\varepsilon \mu_{0}\left(p+\varepsilon \mu_{0} \exp \left(s_{0}-(n+1) \tau\right)\right)} \\
= & M_{n} M_{n+1}\left[1-f\left(s_{0}-(n+1) \tau\right)\right] \\
& +M_{n} M_{n+1}+\frac{|a p| \mathrm{e}^{p \tau} M_{n} M_{n+1}}{\varepsilon \mu_{0}\left(p+\varepsilon \mu_{0} \exp \left(s_{0}-(n+1) \tau\right)\right)}
\end{aligned}
$$

which implies (3.15).

Therefore, to prove (3.7) it suffices to prove that $\prod_{n=1}^{\infty}\left(1+\sigma_{n}\right)$ is convergent, and equivalently to prove that the series $\sum_{n=1}^{\infty} \ln \left(1+\sigma_{n}\right)$ is convergent, and in turn, to show that the improper integral

$$
\int_{1}^{+\infty} \ln \left(2-\frac{|a| \mathrm{e}^{p \tau} \mathrm{e}^{-\tau s}}{p+\varepsilon \mu_{0} \mathrm{e}^{-\tau s}}+\frac{|a p| \mathrm{e}^{p \tau}}{\varepsilon \mu_{0}\left(p+\varepsilon \mu_{0} \mathrm{e}^{-\tau s}\right)}\right) \mathrm{d} s
$$

is convergent. This is true since

$$
\begin{aligned}
& \lim _{s \rightarrow \infty} \frac{\ln \left(2-\frac{|a| \mathrm{e}^{p \tau} \mathrm{e}^{-\tau s}}{p+\varepsilon \mu_{0} \mathrm{e}^{-\tau s}}+\frac{|a p| \mathrm{e}^{p \tau}}{\varepsilon \mu_{0}\left(p+\varepsilon \mu_{0} \mathrm{e}^{-\tau s}\right)}\right)}{\left(1+s^{2}\right)^{-1}} \\
& =\lim _{s \rightarrow \infty} \frac{\frac{\mathrm{d}}{\mathrm{d} s} \ln \left(2-\frac{|a| \mathrm{e}^{p \tau} \mathrm{e}^{-\tau s}}{p+\varepsilon \mu_{0} \mathrm{e}^{-\tau s}}+\frac{|a p| \mathrm{e}^{p \tau}}{\varepsilon \mu_{0}\left(p+\varepsilon \mu_{0} \mathrm{e}^{-\tau s}\right)}\right)}{\frac{\mathrm{d}}{\mathrm{d} x}\left(1+s^{2}\right)^{-1}} \\
& =-\lim _{s \rightarrow \infty}\left(\frac{p \tau|a| \mathrm{e}^{p \tau} \mathrm{e}^{-\tau s}}{\left(p+\varepsilon \mu_{0} \mathrm{e}^{-\tau s}\right)^{2}}+\frac{\varepsilon \mu_{0} \tau|a p| \mathrm{e}^{p \tau} \mathrm{e}^{-\tau s}}{\varepsilon \mu_{0}\left(p+\varepsilon \mu_{0} \mathrm{e}^{-\tau s}\right)^{2}}\right) \frac{\left(1+s^{2}\right)^{2}}{2 s} \\
& =0 .
\end{aligned}
$$

Now, let us prove (3.8). It is sufficient to prove

$$
\max _{0 \leqslant t \leqslant \sigma / \lambda}\|u(t)\| \leqslant C(\varepsilon, a, \lambda)\left\|u_{0}\right\|_{C\left([-\sigma, 0], L^{2}(\Omega)\right)}
$$

since $\ln \frac{\sigma}{1-\lambda} \leqslant s \leqslant \ln \frac{\sigma}{1-\lambda}-\ln \lambda$ is equivalent to $0 \leqslant t=\mathrm{e}^{s}-\frac{\sigma}{1-\lambda} \leqslant \frac{\sigma}{\lambda}$.

Set $\phi(t)=\max _{0 \leqslant s \leqslant t}\|u(s)\|^{2}$ and let $C=C(\varepsilon, a, \lambda)$ denote various positive constants which may be different in different occurrences. Then we have

$$
0=\int_{\Omega} u\left(u_{t}(x, t)-\varepsilon \Delta u(x, t)-a u(x, \lambda t-\sigma)\right) \mathrm{d} x
$$


and hence for any $0 \leqslant t_{1} \leqslant t$

$$
\begin{aligned}
\frac{\mathrm{d}}{\mathrm{d} s}(\|u(s)\|) & \leqslant 2|a| \int_{\Omega} u(s) u(\lambda s-\sigma) \mathrm{d} x \\
& \leqslant C\left\|u_{0}\right\|_{C\left([-\sigma, 0], L^{2}\right)}+C \phi(s)
\end{aligned}
$$

and then

$$
\begin{aligned}
\left\|u\left(t_{1}\right)\right\|^{2} & \leqslant\left(C t_{1}+1\right)\left\|u_{0}\right\|_{C\left([-\sigma, 0], L^{2}\right)}+C \int_{0}^{t_{1}} \phi(s) \mathrm{d} s \\
& \leqslant\left(C t_{1}+1\right)\left\|u_{0}\right\|_{C\left([-\sigma, 0], L^{2}\right)}+C \int_{0}^{t} \phi(s) \mathrm{d} s .
\end{aligned}
$$

Consequently

$$
\phi(t) \leqslant(C t+1)\left\|u_{0}\right\|_{C\left([-\sigma, 0], L^{2}\right)}+C \int_{0}^{t} \phi(s) \mathrm{d} s
$$

which yields by Gronwall's inequality that for any $0 \leqslant t \leqslant \sigma / \lambda$

$$
\phi(t) \leqslant C(\varepsilon, a, \lambda)\left\|u_{0}\right\|_{C\left([-\sigma, 0], L^{2}\right)} .
$$

REMARK 3.3 (i) We guess that the exponent $\frac{1}{\ln \lambda} \ln \left(\frac{\varepsilon \mu_{0}}{|a|}\right)$ given by (3.3) is optimal. That is, if the solution of (1.8)-(1.10) satisfies

$$
\lim _{t \rightarrow+\infty} \frac{\|u(t)\|}{t^{\frac{1}{\ln \lambda} \ln \left(\frac{\varepsilon \mu_{0}}{|a|}\right)}}=0,
$$

then $u(x, t) \equiv 0$ in $\Omega \times \mathbb{R}_{+}$.

(ii) If $|a|<\varepsilon \mu_{0}$, then $\frac{1}{\ln \lambda} \ln \left(\frac{\varepsilon \mu_{0}}{|a|}\right)<0$. Therefore, the solution of (1.8)-(1.10) decays to zero at a polynomial rate as $t \rightarrow+\infty$.

(iii) If we want to compare the case $\lambda=1$ and the case $0<\lambda<1$, let, for simplicity, $\sigma=0$. Then, if $\lambda=1$ and $a>\varepsilon \mu_{0}$, problem (1.8)-(1.10) has an exponentially growing solution $u=\varphi_{0} \exp \left(\left(a-\varepsilon \mu_{0}\right) t\right)$ where $\varphi_{0}$ is the eigenfunction corresponding to $\mu_{0}$. On the other hand, if $0<\lambda<1$, Theorem 3.2 shows that all solutions grow at most polynomially. This means that the proportionally delayed term $u(x, \lambda t)$ has a anti-dissipative effect in the case $0<\varepsilon \mu_{0}$. For $a<\varepsilon \mu_{0}$, if $\lambda=1$, all solutions of (1.8)-(1.10) satisfy

$$
\|u(t)\| \leqslant\left\|u_{0}\right\| \exp \left(\left(a-\varepsilon \mu_{0}\right) t\right)
$$

while the solutions decay probably only polynomially if $0<\lambda<1$. This shows that the proportionally delayed term $u(x, \lambda t)$ has a dissipative effect in the case $a<\varepsilon \mu_{0}$.

\subsection{Asymptotic behaviour of solutions to (1.11)-(1.13)}

In this section we study the problem (1.11)-(1.13). Whether problem (1.11)-(1.13) has a solution is open. However, if it has a solution then we have the following theorem. 
THEOREM 3.4 Assume that $\varepsilon>0,0<\lambda<1$ and $a$ is a real number. Let $p>\frac{1}{\lambda(1-\lambda)}$, then if problem (1.11)-(1.13) has a solution there exists a positive constant $C=C(\varepsilon, a, \lambda)$ such that the solution of (1.11)-(1.13) satisfies

$$
\begin{gathered}
\|u(t)\| \leqslant C\left\|u_{0}\right\|(1+t)^{p}, \quad \forall t \geqslant 0, \\
\left\|u_{t}(t)\right\| \leqslant C\left\|u_{0}\right\|(1+t)^{p-1}, \quad \forall t \geqslant 0 .
\end{gathered}
$$

Proof. We make the change of variables

$$
t=\mathrm{e}^{\mathrm{e}^{s}}, \quad \tau=\ln \lambda<0, \quad v(x, s)=\mathrm{e}^{-p \mathrm{e}^{s}} u\left(x, \mathrm{e}^{\mathrm{e}^{s}}\right) .
$$

The function $v$ satisfies

$$
\begin{aligned}
& v_{s}(x, s)=\varepsilon \mathrm{e}^{s+\mathrm{e}^{s}} \Delta v(x, s)-p \mathrm{e}^{s} v(x, s)+a \mathrm{e}^{s+\mathrm{e}^{s}+(\lambda-1) p \mathrm{e}^{s}} v(x, \tau+s) \\
& \quad \text { in } \quad \Omega \times \mathbb{R}, \\
& v(0, s)=v(1, s)=0 \quad \text { in } \mathbb{R}
\end{aligned}
$$

since

$$
\begin{aligned}
& u_{t}(x, t)=\left(v_{s}(x, s)+p \mathrm{e}^{s} v(x, s)\right) \exp \left((p-1) \mathrm{e}^{s}-s\right) \\
& u\left(x, t^{\lambda}\right)=u\left(x, \mathrm{e}^{\lambda \mathrm{e}^{s}}\right)=\mathrm{e}^{p \lambda \mathrm{e}^{s}} v(x, \tau+s) .
\end{aligned}
$$

As in the proof of Theorem 3.2, to prove (3.20) it suffices to prove that the solution $v$ of (3.22)-(3.23) is bounded. That is, it suffices to prove that

$$
M_{n}:=\max _{-n \tau \leqslant s \leqslant-n \tau-\tau}\|v(s)\|, \quad n=0,1, \ldots
$$

is bounded.

Noting that $\mu_{0}\|v(x, t)\|^{2} \leqslant\|\nabla v(t)\|^{2}$ we have by integration by parts

$$
\begin{aligned}
\frac{\mathrm{d}}{\mathrm{d} s} \int_{\Omega} v^{2}(s) \mathrm{d} x= & 2 \int_{\Omega} v(s) v_{s}(s) \mathrm{d} x \\
= & -2 \varepsilon \exp \left(s+\mathrm{e}^{s}\right) \int_{\Omega}|\nabla v(s)|^{2} \mathrm{~d} x-2 p \mathrm{e}^{s} \int_{\Omega} v^{2}(s) \mathrm{d} x \\
& -2 a \exp \left[s+\mathrm{e}^{s}+(\lambda-1) p \mathrm{e}^{s}\right] \int_{\Omega} v(s) v(s+\tau) \mathrm{d} x \\
\leqslant & -2\left[p \mathrm{e}^{s}+\varepsilon \mu_{0} \exp \left(s+\mathrm{e}^{s}\right)\right] \int_{\Omega}|v(s)|^{2} \mathrm{~d} x \\
& -2 a \exp \left[s+\mathrm{e}^{s}+(\lambda-1) p \mathrm{e}^{s}\right] \int_{\Omega} v(s) v(s+\tau) \mathrm{d} x,
\end{aligned}
$$

and hence

$$
\begin{aligned}
& \frac{\mathrm{d}}{\mathrm{d} s}\left(\exp \left(2 p \mathrm{e}^{s}+2 \varepsilon \mu_{0} \exp \left(\mathrm{e}^{s}\right)\right) \int_{\Omega} v^{2}(s) \mathrm{d} s\right) \\
& \leqslant-2 a \exp \left[2 p \mathrm{e}^{s}+2 \varepsilon \mu_{0} \exp \left(\mathrm{e}^{s}\right)+s+\mathrm{e}^{s}+(\lambda-1) p \mathrm{e}^{s}\right] \int_{\Omega} v(s) v(s+\tau) \mathrm{d} x .
\end{aligned}
$$


The integration of $(3.25)$ over $(-(n+1) \tau, s)$ yields

$$
\begin{aligned}
& {\left[\exp \left(2 p \mathrm{e}^{t}+2 \varepsilon \mu_{0} \exp \left(\mathrm{e}^{t}\right)\right) \int_{\Omega} v^{2}(t) \mathrm{d} x\right]_{-(n+1) \tau}^{s}} \\
& \leqslant-2 a \int_{-(n+1) \tau}^{s} \exp \left[2 p \mathrm{e}^{t}+2 \varepsilon \mu_{0} \exp \left(\mathrm{e}^{t}\right)+t+\mathrm{e}^{t}+(\lambda-1) p \mathrm{e}^{t}\right] \int_{\Omega} v(t) v(t+\tau) \mathrm{d} x \mathrm{~d} t \\
& \leqslant-2 \tau|a| M_{n} M_{n+1} \exp \left[2 p \mathrm{e}^{s}+2 \varepsilon \mu_{0} \exp \left(\mathrm{e}^{s}\right)+s+\mathrm{e}^{s}+(\lambda-1) p \mathrm{e}^{-(n+1) \tau}\right],
\end{aligned}
$$

which implies that

$$
M_{n+1}^{2} \leqslant M_{n}^{2}-2 \tau|a| M_{n} M_{n+1} \exp \left[-(n+2) \tau+\mathrm{e}^{-(n+2) \tau}+(\lambda-1) p \mathrm{e}^{-(n+1) \tau}\right] .
$$

Consequently

$$
M_{n+1} \leqslant\left(1+2|\tau||a| \mathrm{e}^{-\tau} \exp \left[-(n+1) \tau+\delta \mathrm{e}^{-(n+1) \tau}\right]\right) M_{n}
$$

where we set $\delta=\mathrm{e}^{-\tau}+(\lambda-1) p<0$.

Indeed, if $M_{n+1} \leqslant M_{n}$, then (3.27) holds. If $M_{n+1}>M_{n}$, then (3.27) follows from (3.26). Therefore, to prove that $M_{n}$ is bounded, it suffices to prove that the product

$$
\prod_{n=1}^{\infty}\left(1+2|\tau||a| \mathrm{e}^{-\tau} \exp \left[-(n+1) \tau+\delta \mathrm{e}^{-(n+1) \tau}\right]\right)
$$

is convergent, and equivalently to show that the improper integral

$$
\int_{1}^{\infty} \ln \left(1+2|\tau||a| \mathrm{e}^{-\tau} \exp \left[-\tau s+\delta \mathrm{e}^{-\tau s}\right]\right) \mathrm{d} s
$$

is convergent, which is true as in the proof of Theorem 3.2.

\section{Acknowledgements}

The author thanks very much the referee for the valuable remarks and suggestions which greatly improve the clarity and the presentation of the paper.

\section{REFERENCES}

BALL, J. (1977) Remarks on the blow up and nonexistence theorems for nonlinear evolution equations. Quart. J. Math. Oxford, 28, 473-486.

CARLSAw, M. S. (1921) Introduction to the Mathematical Theory of the Conduction of Heat in Solids. London: Macmillan.

Cerami, G., Solimini, S. \& Struwe, M. (1986) Some existence results for superlinear boundary value problems involving critical exponents. J. Funct. Analysis, 69, 289-306.

FILO, J. (1987) On solutions of a perturbed fast diffusion equation. Appl. Math., 32, 364-380.

Fujita, H. (1966) On the blow up of solutions of the Cauchy problem for $u_{t}=\Delta u+u^{1+\alpha} . J$. Fac. Sci. Univ. Tokyo Sect. IA Math., 13, 109-124. 
Fujita, H. (1968) On some nonexistence and nonuniqueness theorems for nonlinear parabolic equations. Nonlinear Functional Analysis. Proc. Sympos. Pure Math. 18. Providence, RI: American Mathmetical Society, pp. 105-124.

Galaktionov, V. A. (1981) Boundary value problem for the nonlinear parabolic equation $u_{t}=$ $\Delta u^{1+\sigma}+u^{\beta+1}$. Differencial'nye Uravnenija, 17, 836-842.

IKeHATA, R. (2000) The Palais-Smale condition for the energy of some semilinear parabolic equations. Hiroshima Math. J., 30, 117-127.

IKeHATA, R. \& SUZUKI, T. (2000) Semilinear parabolic equations involving critical Sobolev exponent: local and asymptotic behaviour of solutions. Differential and Integral Equations, 13, 869-901.

KATO, T. \& MCLEOD, J. B. (1971) The functional-differential equation $y^{\prime}(x)=a y(\lambda x)+b y(x)$. Bull. Amer. Math. Soc., 77, 891-937.

Ladyzhenskaya, O. A., Solonnikov, V. A. \& Ural'ceva, N. N. (1968) Linear and Quasilinear Equations of Parabolic Type. Translations of AMS 23. Providence, RI: American Mathematical Society.

LEvine, H. A. (1973) Some nonexistence and instability theorems for solutions of formally parabolic equations of the form $P u_{t}=-A u+\mathcal{F} u$. Arch. Rational Mech. Anal., 51, 371-386.

LEVINE, H. A. \& SAKS, P. E. (1984) Some existence and nonexistence theorems for solutions of degenerate parabolic equation. J. Diff. Equat., 52, 135-161.

NAKAO, M. (1983) Existence, nonexistence and some asymptotic behaviour of global solutions of a nonlinear degenerate parabolic equation. Math. Rep. Kyushu Univ., 14, 1-21.

Samarskit, A. A., Galaktionov, V. A., Kurdyumov, S. P. \& Mikhailov, A. P. (1995) Blow up in Quasilinear Parabolic Equations de Gruyter Exp. Math., Vol. 19. Berlin: de Gruyter.

STRUwe, M. (1984) A global compactness result for elliptic boundary value problem involving limiting nonlinearities. Math. Z., 187, 511-517.

Tsutsumi, M. (1972) Existence and nonexistence of global solutions for nonlinear parabolic equations. Publ. Res. Inst. Math. Sci., 8, 211-229.

Wu, J. (1996) Theory and Applications of Partial Functional Differential Equations. New York: Springer. 